\title{
Conformal transformation of Dyakonov surface waves into bound- states of cylindrical metamaterials
}

\author{
Veerachart Kajorndejnukul ${ }^{1}$, David Artigas ${ }^{1,2}$, and Lluis Torner ${ }^{1,2}$ \\ 1. ICFO-Institut de Ciències Fotòniques, The Barcelona Institute of Science and Technology, 08860 \\ Castelldefels (Barcelona), Spain. \\ 2. Department of Signal Theory and Communications, Universitat Politecnica de Catalunya, 08034 \\ Barcelona, Spain
}

\begin{abstract}
We put forward the use of transformation optics to map surface waves that exist as one-dimensional modes supported by anisotropic structures into bound states in twodimensional geometries. Specifically, we show the conformal mapping of Dyakonov waves existing in infinite planar surfaces separating birefringent media into bound modes supported by a cylindrical structure made of suitable metamaterials. In contrast with the original Dyakonov waves, the resulting fiber-like modes are highly dispersive, may exist as fundamental as well as higher-order states, feature helical wavefronts, and exhibit a lower and upper frequency cut-off. The program we put forward can be applied to all wave phenomena currently known to occur only in planar geometries in different types of anisotropic media.
\end{abstract}

Transformation optics (TO) is a powerful mathematical method based on the form invariance of Maxwell's equations upon coordinate transformation [1,2]. The transformation relies on the physical equivalence between wave propagation in a curved space and in media with general inhomogeneous and anisotropic properties. It provides an approach to control the flow of light in a material with spatially varying constitutive parameters closely related to a coordinate transformation, enabling the exploration of spectacular effects, such as partial invisibility cloaks [3-7], optical illusion [8,9], the optical analogue of gravitational lensing $[10,11]$ and event horizons around black holes [12], as well as the design of a variety of photonic devices [13]. For example, TO has been applied to the design of optical waveguides and cavities in order to achieve multimode waveguide bends with minimal intermode coupling [14] and highly directional emission of whispering gallery modes [15], respectively, as well as omni-directional optical concentrators for solar applications $[16,17]$, metallic nanostructures for ultra-sensitive spectroscopy [18-20], and nonlocal effects and van der Waals interactions in plasmonic systems [18-20], among other optical phenomena and applications. 
To date, the presence of anisotropy in the original space has been usually avoided in TO analysis in order to simplify the models and, also, the anisotropy of the resulting metamaterials inherently introduced by the transformation is seen as an unwanted side effect [21]. In contrast, here we start from an anisotropic medium with the goal to explore how wave phenomena that occur in planar structures (i.e., where guided waves are described by only one transverse dimension) because of the presence of anisotropy, transform into states guided in cylindrical structures under the conformal mapping. Specifically, we study the conformal transformation of so-called Dyakonov surface waves (DSWs) from one-dimensional states into fiber-like modes.

Dyakonov surface waves are a special type of surface states in the sense that in contrast, e.g., to plasmons, they form in lossless materials. Importantly, material anisotropy is a necessary ingredient for their existence. They were first observed experimentally [22] at the surface of the biaxial birefringent crystal of potassium titanyl phosphate after the original theoretical prediction in 1988 [23]. In their simplest form, Dyakonov surface waves exist at the interface between an isotropic cladding with refractive index $\mathrm{n}_{\mathrm{c}}$, and a uniaxial birefringent substrate with ordinary, $\mathrm{n}_{\mathrm{o}}$, and extraordinary, $\mathrm{n}_{\mathrm{e}}$, refractive indices. When $\mathrm{n}_{\mathrm{o}}<\mathrm{n}_{\mathrm{c}}<\mathrm{n}_{\mathrm{e}}$ and the optical axis (OA) of the birefringent crystal lies on the interface plane, rigorously-bounded (i.e., fully guided) surface waves exist within a narrow angular existence domain $\Delta \theta$ around a central angle $\theta_{0}$. The bound states are always hybrid modes, containing both transverse electric and transverse magnetic waves in the isotropic media, as well as both ordinary and extraordinary waves in the birefringent material.

During the last two decades, the existence and properties of Dyakonov-like surface states have been addressed in a variety of materials, including different dielectric crystals $[22,25]$, negative-index materials [26], magnetic materials [27], chiral materials [28], natural [29] and nanostructured van der Waals materials [30], and general elliptic [31,32] and hyperbolic [33-35] metamaterials. However, studies have been always restricted to planar geometries. Here we make use of TO techniques to address their generalization to settings with higher-dimensional confinement. Our finding reveals that the transformed states exhibit properties reminiscent of the one-dimensional modes and also acquire new characteristics dictated by the transformation. In particular, we show that the transformation enforces a change in the topology of the eigenmodes and introduces new modal dispersion properties. For the transformation, we use the complex exponential mapping function [36], which is conformal, and thus retains the birefringent uniaxial nature of the original media [37], as

$$
\mathrm{w}=\mathrm{Re}^{\frac{\mathrm{z}}{\mathrm{R}}},
$$


where $\mathrm{z}=\mathrm{x}+\mathrm{iy}$ and $\mathrm{w}=\mathrm{u}+\mathrm{iv}$ are complex variables that describe the spatial coordinates in the original and transformed complex spaces, respectively, and $\mathrm{R}$ is a radius that determines the mapping of the surface $\mathrm{x}=0$ to the transformed space. Basically, the mapping connects the horizontal and vertical grids of the Cartesian coordinates $(x, y, Z)$ in the original space [Fig. 1(a)] to concentric circles and radial lines of the cylindrical coordinates $\left(\rho, \phi, Z^{\prime}\right)$ in the transformed space [Fig. 1(b)], respectively, according to the mathematical relationship of $\rho=\mathrm{R}^{\frac{\mathrm{x}}{\mathrm{R}}}$ and $\phi=2 \pi \mathrm{p}+\mathrm{y} / \mathrm{R}$ where $\mathrm{p}$ is an integer so that $\phi \in[0,2 \pi)$ for any value of $y$.

We consider a planar interface located at $\mathrm{x}=0$ between a semi-infinite isotropic medium and a uniaxial crystal with its $\mathrm{OA}$ chosen to be oriented along the $\mathrm{Z}$ direction [Fig. 1(c)]. The refractive indices $\mathrm{n}_{\mathrm{o}}, \mathrm{n}_{\mathrm{e}}$, and $\mathrm{n}_{\mathrm{c}}$ are set to be $1.25,2$, and 1.3 , respectively, resulting in a DSW propagating within the angular range $\Delta \theta=\left(17.8^{\circ}, 24.7^{\circ}\right)$. The electric-field amplitude and phase of the y component of the DSW propagating at an angle $\theta=18.04^{\circ}$ are shown in Figs. $1(\mathrm{e})$ and $1(\mathrm{~g})$, respectively. The plots show that the mode is confined at the interface and that there is a periodic phase variation along the $y$ direction associated with a tilted planar wavefront of the DSW. The spatial periodicity $T_{D}$ of the phase pattern along the $y$ direction is associated with the mode propagation constant through $T_{D}=$ $2 \pi /\left(\mathrm{Nk}_{0} \sin \theta\right)$, where $\mathrm{N}$ is the effective mode index and $\mathrm{k}_{0}$ is the wavenumber in vacuum.

Upon the two-dimensional $\left(Z^{\prime}=Z\right)$ coordinate transformation (1), the infinitely extended planar interface located at $\mathrm{x}=0$ in the original space [Fig. 1(c)] is wrapped in the transformed space into a cylindrical interface located at $\rho=\mathrm{R}$ of a fiber-like structure with a central axis aligned along the $\mathrm{Z}$ direction [Fig. 1(d)]. As a result, the periodicity of the $\phi$ coordinate in the transformed space requires a field periodicity $\mathrm{T}_{\mathrm{T}}=2 \pi \mathrm{R}$ along the $\mathrm{y}$ direction in the original space. To ensure the field existence (constructive interference), $\mathrm{T}_{\mathrm{D}}$ must be a non-zero integer multiple $\mathrm{m}$ of $\mathrm{T}_{\mathrm{T}}$, resulting in the wavelength selection rule for the mode transformation,

$$
\frac{\lambda_{\mathrm{r}}}{\mathrm{R}}=\frac{2 \pi}{\mathrm{m}} \cdot \mathrm{N} \sin \theta \text {. }
$$

Following [38], this equation is therefore the embedding of the transformed configuration onto the original one, which for $\phi=[0,2 \pi)$ covers only a portion of the plane where periodicity is imposed.

At the resonant wavelength $\left(\lambda_{\mathrm{r}}\right)$, the DSW in the original space, which is a plane wave, acquires a topological change after the transformation resulting in a bound state supported by the cylindrical interface with a helical phase front given by $\mathrm{e}^{\mathrm{im} \phi}$, where $\mathrm{m}$ describes a winding number or topological charge associated with a phase twist [m=+4 in Fig. 1(h)]. Note that a DSW propagating at a symmetric angle $(-\theta)$ is transformed into a helical wave with a charge $-m$ under the resonant condition. 
The field amplitude and phase shown in Figs. $1(f)$ and $1(h)$ were obtained by exploiting the connection of the electric and magnetic fields in the original $(\mathbf{E}, \mathbf{H})$ and transformed spaces $\left(\mathbf{E}^{\prime}, \mathbf{H}^{\prime}\right)$ :

$$
\mathbf{E}^{\prime}=\left(\Lambda^{\mathrm{T}}\right)^{-1} \mathbf{E} ; \mathbf{H}^{\prime}=\left(\Lambda^{\mathrm{T}}\right)^{-1} \mathbf{H},
$$

where $\Lambda$ denotes the Jacobian transformation matrix [39], which for our transformation writes:

$$
\Lambda=\left[\begin{array}{lll}
\frac{\partial \mathrm{u}}{\partial \mathrm{x}} & \frac{\partial \mathrm{u}}{\partial \mathrm{y}} & \frac{\partial \mathrm{u}}{\partial \mathrm{Z}} \\
\frac{\partial \mathrm{v}}{\partial \mathrm{x}} & \frac{\partial \mathrm{v}}{\partial \mathrm{y}} & \frac{\partial \mathrm{v}}{\partial \mathrm{z}} \\
\frac{\partial \mathrm{Z}^{\prime}}{\partial \mathrm{x}} & \frac{\partial \mathrm{Z}^{\prime}}{\partial \mathrm{y}} & \frac{\partial \mathrm{Z}^{\prime}}{\partial \mathrm{z}}
\end{array}\right]=\left[\begin{array}{ccc}
\mathrm{e}^{\frac{\mathrm{x}}{\mathrm{R}}} \cos \left(\frac{\mathrm{y}}{\mathrm{R}}\right) & -\mathrm{e}^{\frac{\mathrm{x}}{\mathrm{R}}} \sin \left(\frac{\mathrm{y}}{\mathrm{R}}\right) & 0 \\
\mathrm{e}^{\frac{\mathrm{x}}{\mathrm{R}}} \sin \left(\frac{\mathrm{y}}{\mathrm{R}}\right) & \mathrm{e}^{\frac{\mathrm{x}}{\mathrm{R}}} \cos \left(\frac{\mathrm{y}}{\mathrm{R}}\right) & 0 \\
0 & 0 & 1
\end{array}\right]
$$

Similarly, in the TO formulation, the material permittivity and permeability tensors in the transformed $\left(\varepsilon^{\prime}, \mu^{\prime}\right)$ and the original space $(\varepsilon, \mu)$ are related through:

$$
\varepsilon^{\prime}=\frac{\Lambda \varepsilon \Lambda^{T}}{|\operatorname{det} \Lambda|^{\prime}} ; \mu^{\prime}=\frac{\Lambda \mu \Lambda^{T}}{|\operatorname{det} \Lambda|^{\prime}}
$$

resulting in:

$$
\begin{array}{ccc}
\varepsilon_{\text {core }}=\left[\begin{array}{ccc}
\mathrm{n}_{\mathrm{o}}^{2} & 0 & 0 \\
0 & \mathrm{n}_{\mathrm{o}}^{2} & 0 \\
0 & 0 & \frac{\mathrm{n}_{\mathrm{e}}^{2}}{\rho^{2}}
\end{array}\right], \quad \mu_{\text {core }}=\left[\begin{array}{ccc}
1 & 0 & 0 \\
0 & 1 & 0 \\
0 & 0 & \frac{1}{\rho^{2}}
\end{array}\right] & ; \rho \leq \mathrm{R}, \\
\varepsilon_{\text {cladding }}=\left[\begin{array}{ccc}
\mathrm{n}_{\mathrm{c}}^{2} & 0 & 0 \\
0 & \mathrm{n}_{\mathrm{c}}^{2} & 0 \\
0 & 0 & \frac{\mathrm{n}_{\mathrm{c}}^{2}}{\rho^{2}}
\end{array}\right], \quad \mu_{\text {cladding }}=\mu_{\text {core }} & ; \rho>\mathrm{R} .
\end{array}
$$

Figure 2(a) shows the radial dependence of each element of the permittivity and permeability tensors. A design of a possible practical realization may be obtained by using the effective medium theory [31,32], from which the desired material inhomogeneity and anisotropy may be realized by distributing rods with radially varying diameter, within a unit cell with dimensions $a \ll \lambda$, embedded into a core and a cladding with the same refractive index as the substrate and cladding in the original space. Figure 2(b) shows the rod radius normalized with respect the unit cell $\left(r_{a} / a\right)$ for a particular configuration with hexagonal lattices, composed of magnetic dielectric rods in the core and magnetic metals in the cladding. Such structures may be made at the microwave frequency range by using embedded magnetic ionic liquids [40] or magnetic nanocomposites [41].

To elucidate the existence of generalized Dyakonov-like surface states in the two-dimensional geometry, we used a finite-element (FE) mode solver (Comsol Multiphysics) to find the modes of a cylindrical structure $(\mathrm{R}=1 \mu \mathrm{m})$ with the magneto-dielectric properties given by (6) and (7) at the 
operating wavelength $\lambda_{0}=632.8 \mathrm{~nm}$. Subsequently, we compared the numerical results with the mode obtained by directly transforming the one-dimensional counterpart using (3). When comparing with FE results, note that the numerically computed effective mode index in the cylindrical structure, $\mathrm{N}_{\mathrm{Z}}$, corresponds to the $\mathrm{Z}$ component of the effective index $\mathrm{N}$ of the original $\mathrm{DSW}$, i.e., $\mathrm{N}_{\mathrm{Z}}=\mathrm{N} \cdot \cos \theta$, resulting in $\mathrm{N}_{\mathrm{Z}}=1.236$ for $\theta=18.05^{\circ}$. In addition, the different field components shown in Fig. 3 are associated with the superposition of two helically phased Dyakonov-like states with $m= \pm 4$. The results share a common field structure with small dissimilarities in the vicinity of the center of the core. The minor difference in the mode structure is interpreted as a consequence of the truncation of the infinitely large value of the tensor element $\varepsilon_{\mathrm{zz}}$ and $\mu_{\mathrm{zz}}$ near the center of the core performed in the FE-based calculations.

In contrast to the dispersionless nature of DSWs in the original one-dimensional space, the finite dimension of the cylindrical metamaterial structure in the transformed space introduces a wavelength dependent mode dispersion. As the form invariance of Maxwell equations under coordinate transformation yields the form invariance of the eigenvalue equation, the new dispersion characteristics is a direct consequence of the existence condition (2). This equation establishes the relation between $\theta$ and $\lambda$ at a fixed $m$, so that the angular dispersion $N=f(\theta)$ of the original DSW [see Fig. 4(a)] translates into a wavelength modal dispersion $N_{Z}=N \cos \theta=g(\lambda)$ of the transformed mode [see Fig. 4(b)]. As m varies, different mode branches are formed within a range between a lower $\left(\lambda_{c, \text { min,m}}\right)$ and upper $\left(\lambda_{c, \text { max,m }}\right)$ cut-off wavelength that are related to the lower $\left(\theta_{c, \min }\right)$ and upper $\left(\theta_{c, \text { max }}\right)$ cut-off angle of the original DSW, respectively, so that $m$ represents mode order and charge.

We now examine the mapped field amplitudes to determine whether the transformation affects the existence condition for surface waves. Using (3), the field amplitude at the core of the metamaterial cylinder can be written in a compact form as:

$$
\left\{\begin{array}{c}
E_{\rho}=C_{\rho, o} \rho^{\gamma_{0} k_{0} R-2}+C_{\rho, e} \rho^{\gamma_{e} k_{0} R-2} \\
E_{\phi}=C_{\phi, o} \rho^{\gamma_{0} k_{0} R-2}+C_{\phi, e} \rho^{\gamma_{e} k_{0} R-2} \\
E_{z}=C_{z, o} \rho^{\gamma_{0} k_{0} R}+C_{z, e} \rho^{\gamma_{e} k_{0} R}
\end{array}\right.
$$

where $\mathrm{C}_{\mathrm{i}, \mathrm{o}}$ and $\mathrm{C}_{\mathrm{i}, \mathrm{e}}$ are the amplitude coefficients, and $\gamma_{\mathrm{o}}$ and $\gamma_{\mathrm{e}}$ are the transverse decay constants of the ordinary (o) and extraordinary (e) evanescent waves in the birefringent substrate of the original one-dimensional DSW. Surface guiding requires the power of $\rho$ in (8) to be positive in the core. Since $0<\gamma_{\mathrm{e}}<\gamma_{\mathrm{o}}$ for the original one-dimensional DSW [23], the condition $\gamma_{\mathrm{e}} \mathrm{k}_{0} \mathrm{R}>0$ for $\mathrm{E}_{\mathrm{z}}$ is always fulfilled. In turn, the same condition for transversal components $E_{\rho}$ and $E_{\phi}$ require $\gamma_{\mathrm{e}} \mathrm{k}_{0} \mathrm{R}-2>0$ for surface guiding, which establishes the threshold condition: 


$$
\frac{\lambda_{0}}{\mathrm{R}}<\pi \gamma_{\mathrm{e}}
$$

This condition results in a wavelength upper bound for surface guiding of Dyakonov-like modes, denoted by the orange dashed line in Fig. 4(b). When the condition (9) is met, the fields $E_{\rho}$ and $E_{\phi}$ are localized at the cylindrical interface [see Fig.4(c)]. When (9) is unmet, the one-dimensional DSW in the original space is mapped into field components $E_{\rho}$ and $E_{\phi}$ highly localized at the core center in the transformed space [see Fig. 4 (d)] due to the infinite value of the tensor element $\varepsilon_{z z}$ and $\mu_{z z}$ at this location [Fig. 2(a)]. This phenomenon can happen in each mode branch by either transforming loosely confined modes (low $\gamma_{\mathrm{e}}$ ) or decreasing the effective optical size of the core (small $\mathrm{R} / \lambda_{0}$ ). An important physical consequence of this effect is that lower order modes [ $m=1,2$ in Fig. $4(b)]$ do not exist as surface waves even for metamaterial fibers with relatively large $R / \lambda_{0}$.

Similarly, the transversal field amplitudes in the transformed cladding region are characterized by the factor $\rho^{-\gamma_{c} k_{0} R-2}$, where $\gamma_{c}$ is the transverse decay constant in the cladding of the original DSW, with $\gamma_{c}>0$. In this case, the condition $-\gamma_{c} k_{0} R-2<0$ is always fulfilled and the field mapping into the cladding does not enforce any additional condition for surface guiding.

An important physical result, revealed by Fig. 4(b), is that the number of helical surface modes (existing above the orange dashed line) is either zero or one within the wavelength range of $0.49 \mu \mathrm{m}-0.84 \mu \mathrm{m}$, whereas at shorter $\lambda$ the different mode branches start to overlap and the number of modes can be greater than one. To prevent multi-mode existence over a broader wavelength range, the birefringence of the substrate can be reduced to further limit an angular range of propagation of the one-dimensional DSW leading to a narrower existence range of the two-dimensional generalized DSW in the cylindrical metamaterial and thus well-separated mode branches. The existence of an upper and lower cut-off for each mode allows for the broadband single-mode operation of high order modes in this novel guiding structure.

Next, we study how the transformation affects the mode confinement of the two-dimensional bound modes. We define the localization length (L) as the full-width at half-maximum (FWHM). The localization length at FWHM for the y component of the one-dimensional DSW, $L_{0}$, is estimated to be $14 \lambda_{0}$ for the original planar structure used in Fig. $1(\mathrm{e})$ and $\theta=18.04^{\circ}$. The angular field component of the transformed DSW, $E_{\phi}$, obtained by direct field mapping is chosen for the localization measurement because of both its relatively large field amplitude (it is mainly related to $\mathrm{E}_{\mathrm{y}}$ in the original space) and simply characterized mode profile. Figure 5 shows the normalized localization length $\mathrm{L} / \mathrm{L}_{\mathrm{o}}$ as a function of the normalized core radius $\mathrm{R} / \lambda_{0}$ associated with the transformed modes with $\mathrm{N}_{\mathrm{Z}}=1.236$ and mode order $\mathrm{m}$ being an integer multiple of 4 [according to (2)]. The results 
indicate that the two-dimensional surface modes of the cylindrical metamaterial structure exhibit a tunable and higher localization of light. For instance, at $\mathrm{R} / \lambda_{0}=1.58$ the localization is $\sim 8$ times smaller than $\mathrm{L}_{\mathrm{o}}$.

As mentioned above, the original DSW is mapped into the two-dimensional surface mode when the core radius is above the threshold value given by (9) (for our example, $\mathrm{R}_{\mathrm{th}} / \lambda_{0}=1 / \pi \gamma_{\mathrm{e}} \sim 1.17$ ). Then, the non-uniformly distributed and highly dense coordinate mesh in the vicinity of the central core governed by the exponential transformation [see Fig. 1(b)] facilitates the mapping of the spatially extended DSWs between the one- and the two-dimensional spaces, resulting in a higher degree of localization of the two-dimensional DSW. Physically, the index guiding shown in Fig. 2(a) enables the energy redistribution of the spatially extended one-dimensional DSW to the core of the twodimensional geometry. As the core radius increases, the effect of index guiding is progressively reduced leading to an increase in localization length. As $R / \lambda_{0} \rightarrow \infty$, the localization of the twodimensional modes resembles the one of the one-dimensional DSWs $\left(L / L_{0} \rightarrow 1\right)$ because the spatial variation of the material properties associated with the coordinate transformation is negligible within the wave extension near the interface.

In summary, we have shown that applying a complex conformal exponential mapping, onedimensional Dyakonov surface guided modes that exist in anisotropic media transform into generalized two-dimensional helical waves supported by a cylindrical surface of a metamaterial with transformed electric and magnetic properties. The transformed, two-dimensional modes feature a richer topology, new dispersion properties, upper and lower cut-offs for each mode branch and a tunable localization degree. Fabrication of the cylindrical metamaterial that accompanies the conformal transformation appears to be challenging, as it requires engineered electric and magnetic properties. However, such metamaterials may be achievable by using embedded rods with magnetic and metallo-dielectric properties into a suitable dielectric host. From a broader point of view, we stress that the concept we have put forward here motivates the generalization to higher-dimensional geometries of other types of phenomena that are known to exist only in one-dimensional settings and where anisotropy - of different types - is a necessary ingredient. Salient examples of high current topical interest include hyperbolic plasmons [42], modes of photonic crystal slabs [43], anisotropyinduced bound states in the continuum [44] and metasurfaces [45].

This work has been suported by the Generalitat de Catalunya, CERCA Programme and AGAUR 2017SGR-1400; The Ministry of Economy and Competitiveness of Spain Grant No. FIS2015-71559-P and Severo Ochoa 2016-2019 Grant No. SEV-2015-0522; Fundació Cellex and Fundació Mir-Puig; the 
ICFOstep-stone programme, funded by the H2020 Marie Skłodowska-Curie Actions Cofund Grant No. GA665884 of the European Commission.

\section{References}

[1] J. B. Pendry, D. Schurig, and D. R. Smith, Science 312, 1780 (2006).

[2] U. Leonhardt, Science 312, 1777 (2006).

[3] D. Schurig, J. J. Mock, B. J. Justice, S. A. Cummer, J. B. Pendry, A. F. Starr, and D. R. Smith, Science 314, 977 (2006).

[4] W. Cai, U. K. Chettiar, A. V. Kildishev, and V. M. Shalaev, Nat. Photonics 1, 224 (2007).

[5] J. Valentine, J. Li, T. Zentgraf, G. Bartal, and X. Zhang, Nat. Material 8, 568 (2009).

[6] L. H. Gabrielli, J. Cardenas, C. B. Poitras, and M. Lipson, Nat. Photonics 3, 461 (2009).

[7] T. Ergin, N. Stenger, P. Brenner, J. B. Pendry, and M. Wegener, Science 328, 337 (2010).

[8] Y. Lai, J. Ng, H. Y. Chen, D. Z. Han, J. J. Xiao, Z. Q. Zhang, and C. T. Chan, Phys. Rev. Lett. 102, 253902 (2009).

[9] C. Li, X. Meng, X. Liu, F. Li, G. Fang, H. Chen, and C. T. Chan, Phys. Rev. Lett. 105, 233906 (2010).

[10] D. A. Genov, S. Zhang, and X. Zhang, Nat. Physics 5, 687 (2009).

[11] C. Sheng, H. Liu, Y. Wang, S. N. Zhu, and D. A. Genov, Nat. Photonics 7, 902 (2013).

[12] T. G. Philbin, C. Kuklewicz, S. Robertson, S. Hill, F. König, and U. Leonhardt, Science 319, 1367 (2008).

[13] Q. Wu, J. P. Turpin, and D. H. Werner, Light Sci. Appl. 1, e38 (2012).

[14] L. H. Gabrielli, D. Liu, S. G. Johnson, and M. Lipson, Nat. Commun. 3, 1217 (2012).

[15] Y. Kim, S.-Y. Lee, J.-W. Ryu, I. Kim, J.-H. Han, H.-S. Tae, M. Choi, and B. Min, Nat. Photonics 10, 647 (2016).

[16] A. V. Kildishev and V. M. Shalaev, Opt. Lett. 33, 43 (2008).

[17] M. Rahm, D. Schurig, D. A. Roberts, S. A. Cummer, D. R. Smith, and J. B. Pendry, Photon. Nanostr. Fundam. Appl. 6, 87 (2008).

[18] J. B. Pendry, A. Aubry, D. R. Smith, and S. A. Maier, Science 337, 549 (2012).

[19] J. B. Pendry, A. I. Fernández-Domínguez, Y. Luo, and R. Zhao, Nat. Phys. 9, 518 (2013).

[20] J. B. Pendry, Y. Luo, and R. Zhao, Science 348, 521 (2015).

[21] A. J. Danner, T. Tyc, and U. Leonhardt, Nat. Photonics 5, 357 (2011).

[22] O. Takayama, L. Crasovan, D. Artigas, and L. Torner, Phys. Rev. Lett. 102, 043903 (2009).

[23] M. I. Dyakonov, Sov. Phys. JETP 67, 714 (1988). 
[24] O. Takayama, L.-C. Crasovan, S. K. r. Johansen, D. Mihalache, D. Artigas, and L. Torner, Electromagnetics 28, 126 (2008).

[25] O. Takayama, D. Artigas, and L. Torner, Nat. Nanotechnol. 9, 419 (2014).

[26] L.-C. Crasovan, O. Takayama, D. Artigas, S. K. Johansen, D. Mihalache, and L. Torner, Phys. Rev. B 74, 155120 (2006).

[27] L. C. Crasovan, D. Artigas, D. Mihalache, and L. Torner, Opt. Lett. 30, 3075 (2005).

[28] D. P. Pulsifer, M. Faryad, and A. Lakhtakia, Phys. Rev. Lett. 111, 243902 (2013).

[29] W. Ma, P. Alonso-González, S. Li, A. Y. Nikitin, J. Yuan, J. Martín-Sánchez, J. Taboada-Gutiérrez, I. Amenabar, P. Li, S. Vélez, C. Tollan, Z. Dai, Y. Zhang, S. Sriram, K. Kalantar-Zadeh, S.-T. Lee, R. Hillenbrand, and Q. Bao, Nature 562, 557 (2018).

[30] P. Li, I. Dolado, F. J. Alfaro-Mozaz, F. Casanova, L. E. Hueso, S. Liu, J. H. Edgar, A. Y. Nikitin, S. Vélez, and R. Hillenbrand, Science 359, 892 (2018).

[31] D. Artigas and L. Torner, Phys. Rev. Lett. 94, 013901 (2005).

[32] O. Takayama, D. Artigas, and L. Torner, Opt. Lett. 37, 4311 (2012).

[33] Z. Jacob and E. E. Narimanov, Appl. Phys. Lett. 93, 221109 (2008).

[34] C. J. Zapata-Rodríguez, J. J. Miret, S. Vuković, and M. R. Belić, Opt. Express 21, 19113 (2013).

[35] O. Takayama, E. Shkondin, A. Bodganov, M. E. Aryaee Panah, K. Golenitskii, P. Dmitriev, T. Repaän, R. Malureanu, P. Belov, F. Jensen, and A. V. Lavrinenko, ACS Photon. 4, 2899 (2017).

[36] P. Moon and D. E. Spencer, Field Theory Handbook (Springer-Verlag, Berlin, Heidelberg, 1971).

[37] L. Xu and H. Chen, Nat. Photonics 9, 15 (2014).

[38] R. T. Thompson, S. A. Cummer, and J. Frauendiener, J. Opt. 13, 024008 (2011).

[39] T. J. Cui, D. R. Smith, and R. Liu, Metamaterials: Theory, Design and Applications (Springer, New York, 2010).

[40] S. Hayashi and H. Hamaguchi, Chem. Lett. 33, 1590 (2004).

[41] S. Behrens, Nanoscale 3, 877 (2011).

[42] E. Yoxall, M. Schnell, A. Y. Nikitin, O. Txoperena, A. Woessner, M. B. Lundeberg, F. Casanova, L. E. Hueso, F. H. L. Koppens, and R. Hillenbrand, Nat. Photonics 9, 674 (2015)

[43] C. W. Hsu, B. Zhen, J. Lee, S.-L. Chua, S. G. Johnson, J. D. Joannopoulos and M. Soljačić, Nature 499, 188 (2013).

[44] J. Gomis-Bresco, D. Artigas and L. Torner, Nat. Photonics 11, 232 (2017).

[45] A. V. Kildishev, A. Boltasseva, and V. M. Shalaev, Science 339, 1232009 (2013). 
Original space $(\mathrm{x}, \mathrm{y}, \mathrm{Z})$

(a)

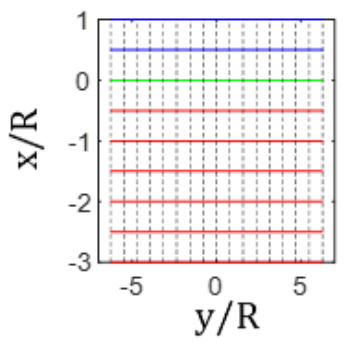

(c)

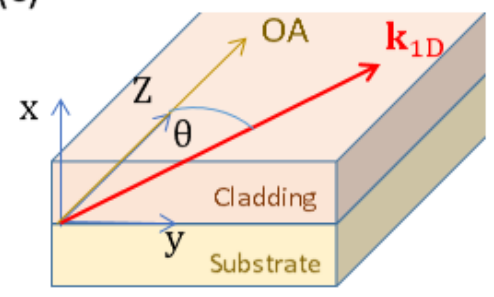

(e)

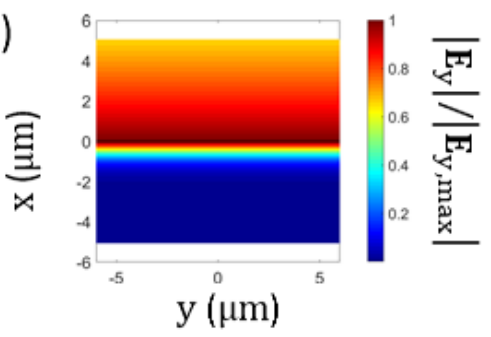

(g)

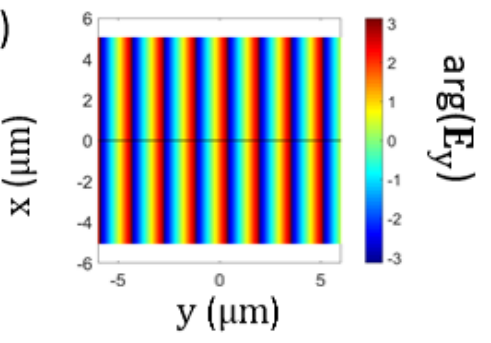

Transformed space $\left(\rho, \phi, Z^{\prime}\right)$

(b)
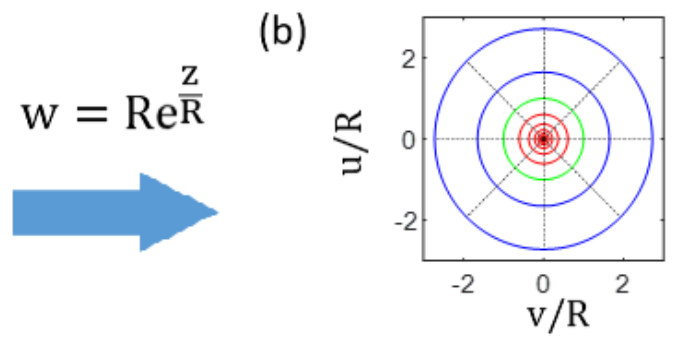

(d)

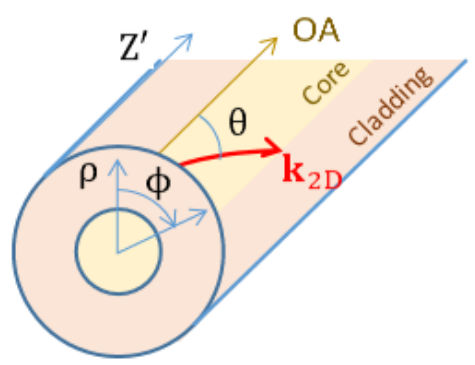

(f)

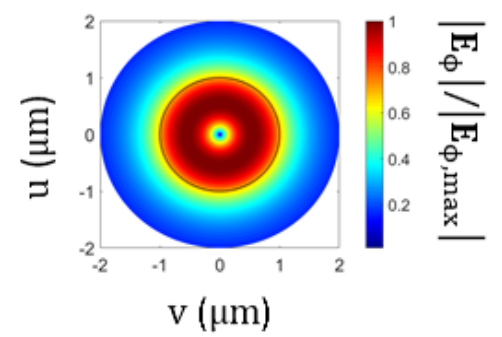

(h)

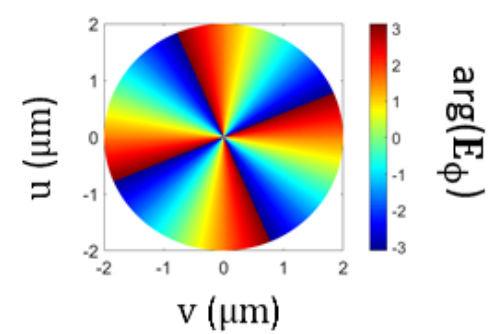

FIG. 1. Conformal transformation of one-dimensional surface states. (a) and (b) conformal map used to transform DSWs existing at planar interfaces of an anisotropic substrate and suitable isotropic cladding (c) into bounds modes existing at the interface of a cylindrical metamaterial (d). Amplitude (e) and phase (g) of the original DSW mapped into an amplitude (f) and helical phase (h) with charge $\mathrm{m}=-4$ in the transformed space. 

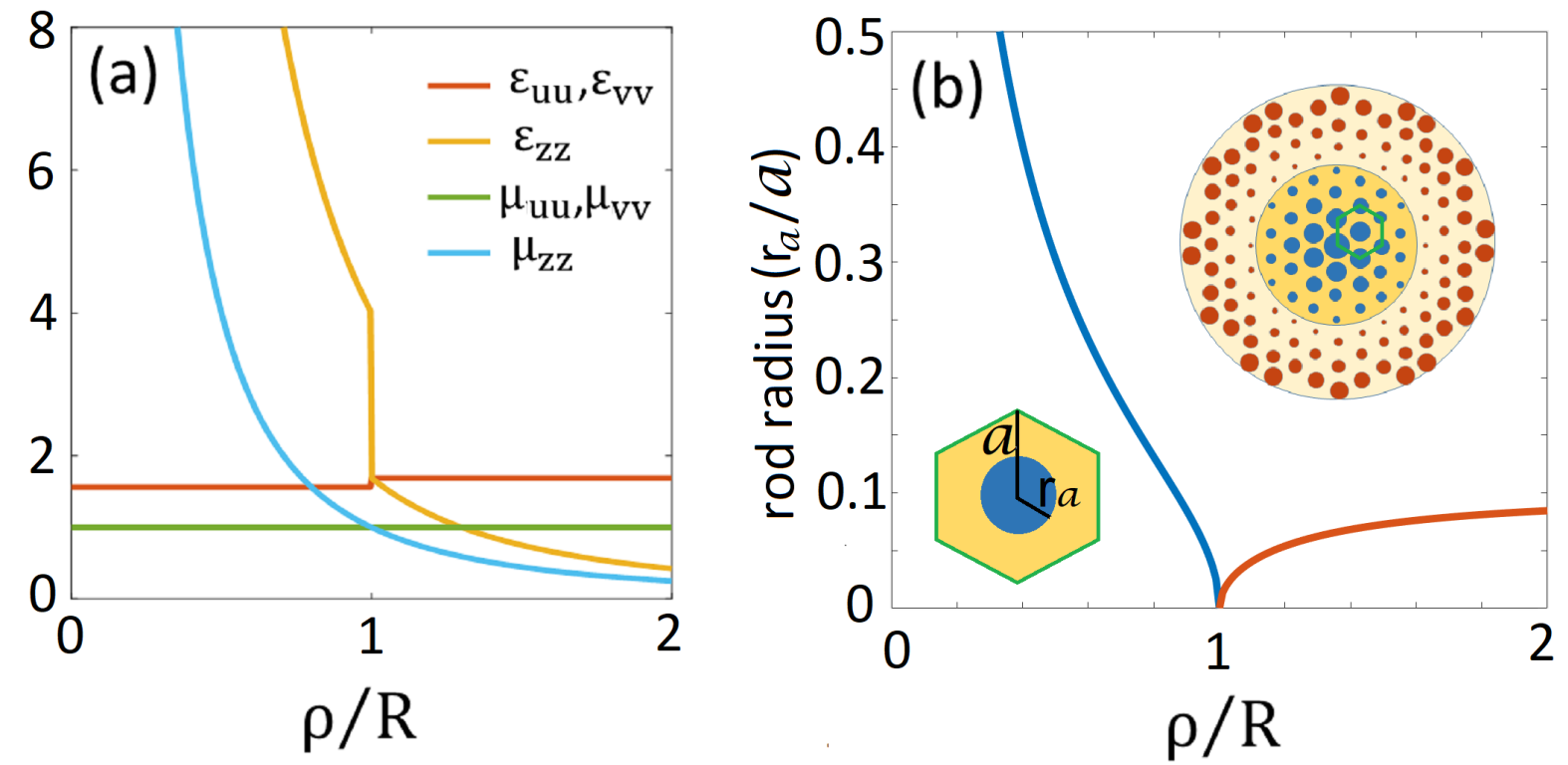

FIG. 2. (a) Radial distribution of the relative permittivity and permeability tensor elements of the cylindrical metamaterial obtained by using Eqs. (6) and (7), with $\mathrm{n}_{\mathrm{o}}=1.25, \mathrm{n}_{\mathrm{e}}=2$, and $\mathrm{n}_{\mathrm{c}}=1.3$ in the original space. (b) The inset shows a potential practical realization constructed by distributing rods with radius $r_{a}$ in a hexagonal lattice with unit cell dimension $a$ made with different electric and magnetic properties in the core and cladding regions with a radially-varying filling factor embedded in a host dielectric core and cladding with the same refractive indices as in the original space. The changing radius of the rods, normalized with respect the cell unit size, are shown as a blue line, corresponding to rods composed of paramagnetic dielectrics with $\mu_{\mathrm{r}}=6$ and $\varepsilon_{\mathrm{r}}=20$. The changing rods radius in the cladding is shown as red lines, and corresponds to rods composed of diamagnetic metals with $\mu_{\mathrm{r}}=-10$ and $\varepsilon_{\mathrm{r}}=-20$. 


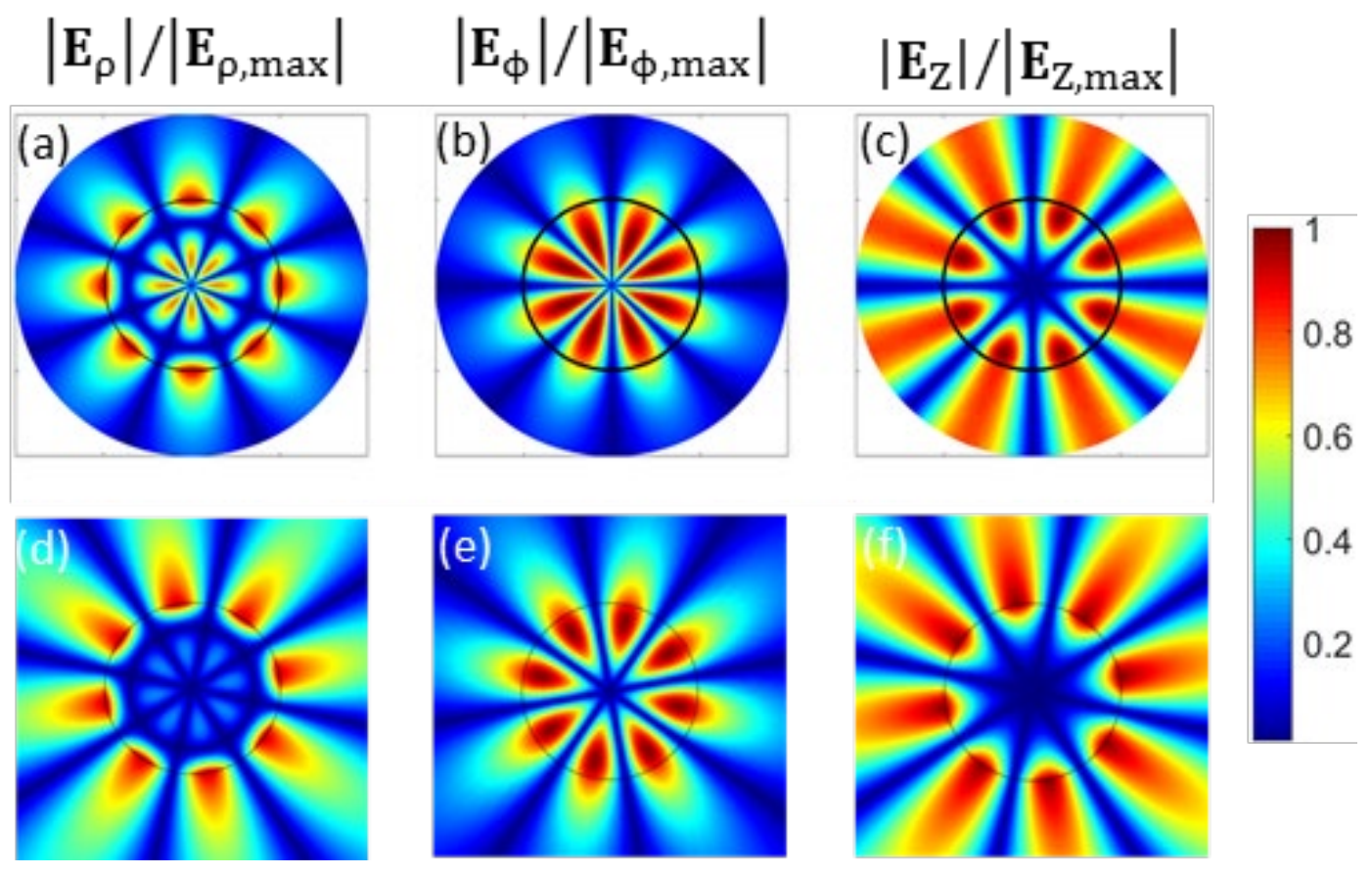

FIG. 3. Electric-field amplitudes of the generalized two-dimensional Dyakonov-like surface states. Electric-field amplitudes of each component, (a) $E_{\rho}$, (b) $E_{\phi}$ and (c) $E_{Z}$, obtained by direct field mapping described by (3). (d)-(f) numerical FE results. The black circular line represents the interface between the core and the cladding regions located at $\rho=R=1 \mu \mathrm{m}$. The comparison between the two sets of results was performed over an equally sized window. 

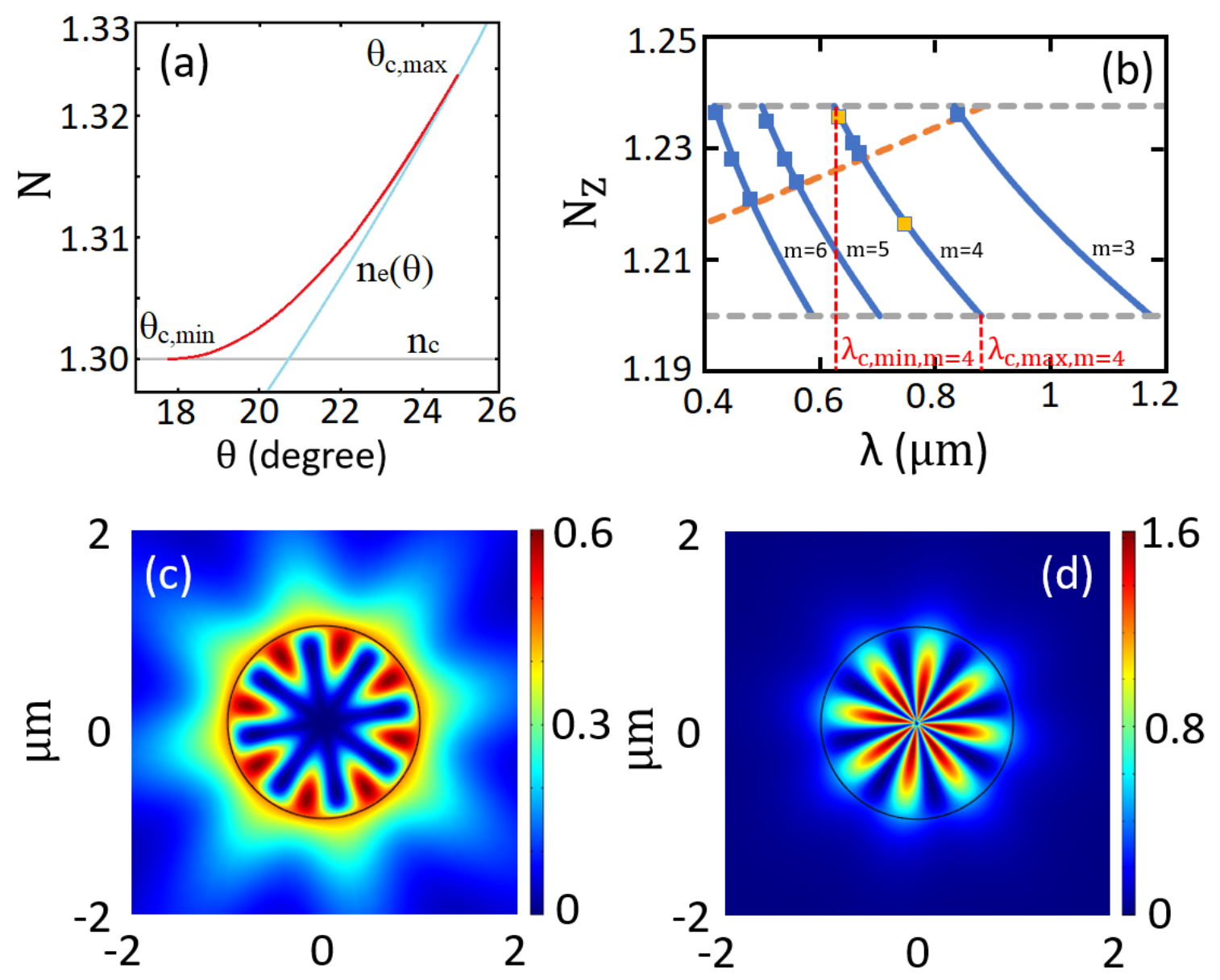

FIG. 4. Mode dispersion of the two-dimensional generalized Dyakonov-like modes. (a) Angular dispersion for the effective index $N$ of the original DSW. The lower $\left(\theta_{c, \min }\right)$ and upper $\left(\theta_{c, \text { max }}\right)$ cut-off angles indicate angular positions for radiation into the cladding $\left(\gamma_{c}=0\right)$ and substrate $\left(\gamma_{e}=0\right)$, respectively. (b) Wavelength dispersion of the transformed DSW showing the effective index, $\mathrm{N}_{\mathrm{Z}}$, for different modes with order $\mathrm{m}$ (blue solid line). Blue markers indicate FE results. The grey dashed lines represent the lower and upper cut-off wavelengths, $\lambda_{c, m i n, m}$ and $\lambda_{c, m a x, m}$, which are related with $\theta_{c, \text { min }}$ and $\theta_{c, \text { max }}$, respectively, through (2). The orange dashed line denotes the threshold for surface guiding. At wavelength above the orange line, DSWs in the original space are mapped into highly localized field at the core center. Power flow calculated with the FE of two EM modes (a) below $(\lambda=$ $0.633 \mu \mathrm{m})$ and $(\mathrm{b})$ above $(\lambda=0.75 \mu \mathrm{m})$ the threshold for surface guiding (indicated by the gold markers in (b)). 


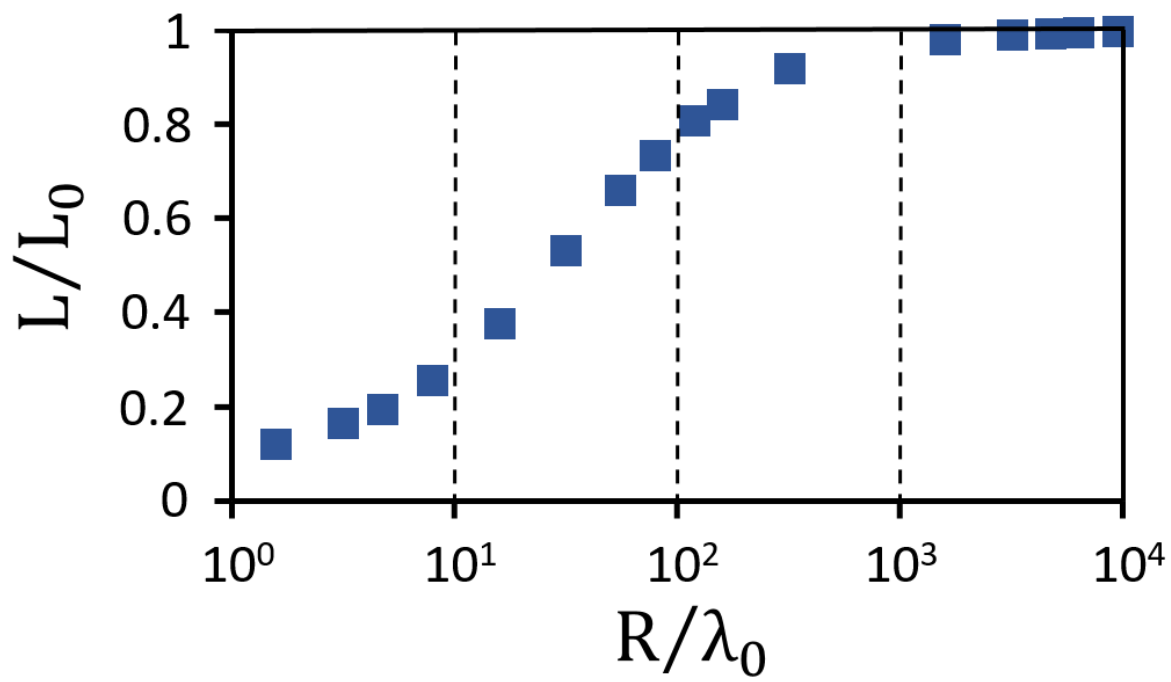

FIG. 5. Normalized localization length $\mathrm{L} / \mathrm{L}_{\mathrm{o}}$ of the two-dimensional generalized Dyakonov-like states as a function of a normalized core radius $\mathrm{R} / \lambda_{0}$ of the cylindrical structure. 